relevant factors. The book ends with two good chapters from North America by DiMatteo and Thorne, respectively. Both embed the subject of non-compliance within the larger contexts of medical treatment of chronic disease in general and of the doctorpatient interaction. They point the way to more fulfilling doctor-patient relationships and greater acceptance by patients of credible offers of treatments.

The book is a useful addition to the still sparse literature addressing the stigmatisation of people with schizophrenia.

Arthur Crisp Emeritus Professor of Psychological Medicine, University of London, Department of General Psychiatry, St George's Hospital Medical School, London SWI7 ORE

\section{Psychiatry and the Human Condition}

By Bruce Charlton. Oxford: Radcliffe Medical Press. 2000.250 pp. $€ 19.95$ (pb).

ISBN I 857753143

I have never met Dr Charlton but he is clearly an interesting and unusual man. After qualifying in medicine and a couple of junior psychiatric posts and a spell in the Medical Research Council's Neuroendocrinology Unit in Newcastle he took an MA in English literature and then worked in succession as a demonstrator in physiology, a lecturer in anatomy and a lecturer in epidemiology and public health before coming to rest, at least temporarily, as a lecturer in psychology.
In this book, which seems to have been written mainly for a lay, or at least a nonmedical audience, he sets out to reinterpret psychiatric disorders and their treatment in the light of evolutionary psychology and cognitive neuroscience. This is potentially a fruitful approach and he starts by suggesting, very plausibly, that delusional disorders (Kraepelin's paranoia) are what he calls "theory of mind delusions", arising not from defective reasoning but from erroneous assumptions about the motives and intentions of other people. Unfortunately, most of what follows is much less plausible. All other delusions, and hallucinations too, are, Charlton suggests, manifestations of delirium, although this has not been recognised by psychiatrists because "disorientation and clouding of consciousness are not sufficiently sensitive measures of delirium". $\mathrm{He}$ goes on to suggest that acute schizophrenia and mania are both fundamentally delirious states, the former superimposed on a progressive brain disease or dementia and the latter precipitated by sleep deprivation. Major depression, however, is not a mood disorder at all but simply the behavioural consequence of an adaptive state of malaise, called "sickness behaviour" in animals, caused primarily by cytokine release. The clinical evidence for the causal role of cytokines is described as "compelling" and the apparent absence of pyrexia in depressive illnesses is attributed to the fact that its presence has "not been evaluated".

Charlton's views about the mode of action of psychiatric drugs are equally novel and even more implausible. Electroconvulsive therapy works by simulating or inducing natural, restorative sleep, which relieves the delirium of mania and acute schizophrenia and presumably of melancholia as well. Most antidepressants are really "antimalaise analgesics" and their effects on mood incidental. The primary role of most neuroleptics is to induce muscular rigidity, which produces a helpful emotional blunting. The therapeutic effects of the atypical neuroleptics are quite different, however, and depend mainly on their "excellent hypnotic properties", while those of clozapine are probably due simply to withdrawal of other neuroleptics and a consequent loss of drug-induced negative symptoms.

Although Charlton has obviously read widely and been strongly influenced by the work of David Healy and Antonio Damasio, he is handicapped by his superficial understanding of the basic phenomena of psychiatric illness and his ignorance, or perhaps dismissal, of contemporary biological and psychological research. Certainly, he makes no attempt to incorporate the findings of genetic or functional imaging research, or even Frith's explorations of delusional ideation, into his speculations. There is at least one genuinely interesting idea here to compensate for the half-baked assertions about aetiology and pharmacology, and the two lengthy appendices about the development of awareness, consciousness, language and creativity are more soundly based than the speculations about mental illness, but overall this book is a disappointment.

Robert Kendell Department of Psychiatry Edinburgh University, 3 West Castle Road, Edinburgh EHI0 5AT 